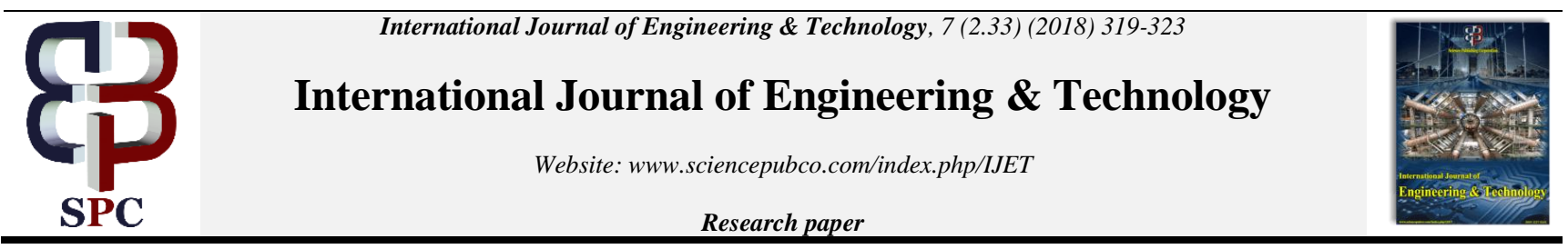

\title{
An analytical study on sliding mode strength at the bonded in- terface depending on the inclined angle of unidirectional CFRP inclined double cantilever with laminated structure
}

\author{
Jae Woong Park ${ }^{1}$, Jae-Ung Cho ${ }^{2} *$ \\ ${ }^{1}$ Department of Mechanical Engineering, Kongju National University, 1223-24, Cheonandaero, Seobuk-GU, Cheonan- \\ SI Chungnam, 31080, Korea \\ ${ }^{2}$ Division of Mechanical \& Automotive Engineering, Kongju National University, 1223-24, Cheonan Daero, Seobuk-gu, Cheonan- \\ SI Chungnam, 31080, Korea \\ *Corresponding author E-mail: jucho@kongju.ac.kr
}

\begin{abstract}
Background/Objectives: CFRP is getting a great deal of attention with its next-generation lightweight materials. Weight lightening can be greatly improved by designing the structure using the method of bonding lightweight materials.

Methods/Statistical analysis: This study was based on BS (British standard) 7991 for mode II (Sliding mode). BS 7991 is a basic research method for identifying the basic elements of CZM (Cohesive zone method). In the mode II as sliding mode of the inclined double cantilever beam, the effects on the materials and the mechanical properties shall be identified when the sliding load working at the bonded interface appears.

Findings: Unidirectional CFRP shall be designed in accordance with quasi-isotropic laminate in order for its elastic stiffness to be designed similarly at all the coordination directions. Therefore, the laminate structure used in this study was designed to be [0/ \pm 603$] \mathrm{s}$. This is because the adhesion force acting on the bonded interface varies according to the progress of the failure when the interfacial failure occurs in the mode II as sliding mode of the inclined double cantilever. Quasi-isotropic laminate structure has an advantage of not setting differently elastic stiffness depending on the coordination in all the directions. The analysis condition of a restraint has been applied to one of the inclined double cantilevers with remote displacement excluding the rotation direction. The freedom of rotation direction along with forced displacement of sliding direction has been given to remote displacement in the other cantilever. Forced displacement speed was set at $3 \mathrm{~mm} / \mathrm{min}$. Given the free direction of rotation in both cantilevers, the inclined double cantilever has been ruptured, minimizing the interference between the elements.

Improvements/Applications: In this research, a database for the application of lightweight materials and the bonding method, which are the design elements for lightweight structure, has been constructed.
\end{abstract}

Keywords: Notched Tensile Specimen; Material; Mechanical Characteristic; Maximum Load; Durability.

\section{Introduction}

In the transportation machinery industry including the automobiles, researches on environment-friendliness have been actively conducted. Most transportation machines are powered by internal combustion engines using fossil fuels [1-3]. Internal combustion engines inevitably create the emissions and have a fatal impact on the environment. Modern technology for transportation has focused on improving fuel consumption and reducing emission $\mathrm{s}$ [4], [5]. However, in the future, many efforts are being made to commercialize the power sources, which do not create emissions using the electric power sources [6]. The cruising range is important for the transportation machine using the electric power source [7-8]. The cruising range of transportation machines using electric power [9-10] directly affects the capacity of battery. The current technology does not meet the requirements for the cruising range and researches on this issue have been actively conducted [11-12]. In terms of current technology improvement and future technology prospects, weight reduction must be indispensable for transportation machines. Reducing the weight of the machine structure can not only improve fuel consumption but also greatly reduce the amount of exhaust gases. In addition, it can significantly increase the cruising range of transportation machines using the electric power. Accordingly, this study provides the basic data for the design of lightweight structures using unidirectional CFRP, which is known as a light material, and adhesion technology.

\section{Analysis models}

Figure 1 shows the specifications of the model used in this study. This study is about mode II (Sliding mode) based on BS (British standard) 7991 showing the test piece of TDCB (Tapered double cantilever beam) type. An angle was given depending on the variable between the models. Research models with tilt angles of $6^{\circ}$, $8^{\circ}, 10^{\circ}$ and $12^{\circ}$ were respectively defined as TDCB 6 , TDCB 8 , TDCB 10, and TDCB 12. All the research models are the same 
with the length of the bonded interface of $120 \mathrm{~mm}$, this being due to a factor which may affect the adhesive force, pertaining to the area of the bonded interface. All the test pieces are the same with the thickness of $5 \mathrm{~mm}$. Figure 1 shows only one cantilever beam of double cantilever beam, which is the research model, being adhered symmetrically to each other with reference to the adherend. Figure 2 shows the structure in the direction of the fiber for analyzing and designing the unidirectional CFRP (Carbon fiber reinforced plastic), a fiber structure composite material. Figure 2 shows the unidirectional CFRP with laminate structure of $\left[0 / \pm 60_{3}\right.$ ]s and with fiber structures of $-60^{\circ}$ and $60^{\circ}$. With the reference to coordination direction, $\mathrm{Z}$-axis direction being $0^{\circ}$ and to $\mathrm{Z}$-axis direction being $\pm 60^{\circ}$, the fibers of $\pm 60^{\circ}$ are laminated, which were designed using the tool called ANSYS Composite Preppost. Also, it shows the adhesive zone as an analytical model after all laminating is completed.

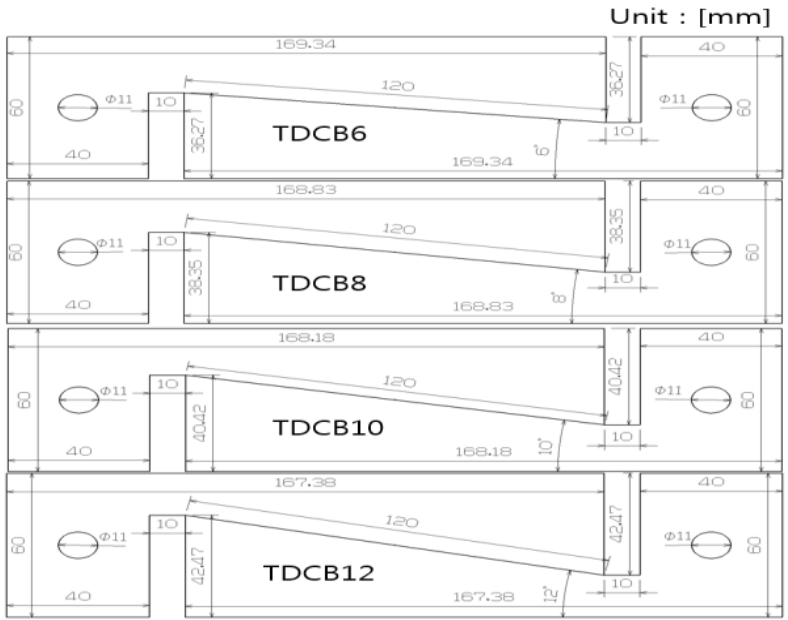

Fig. 1: Design Drawing for Analysis Model.
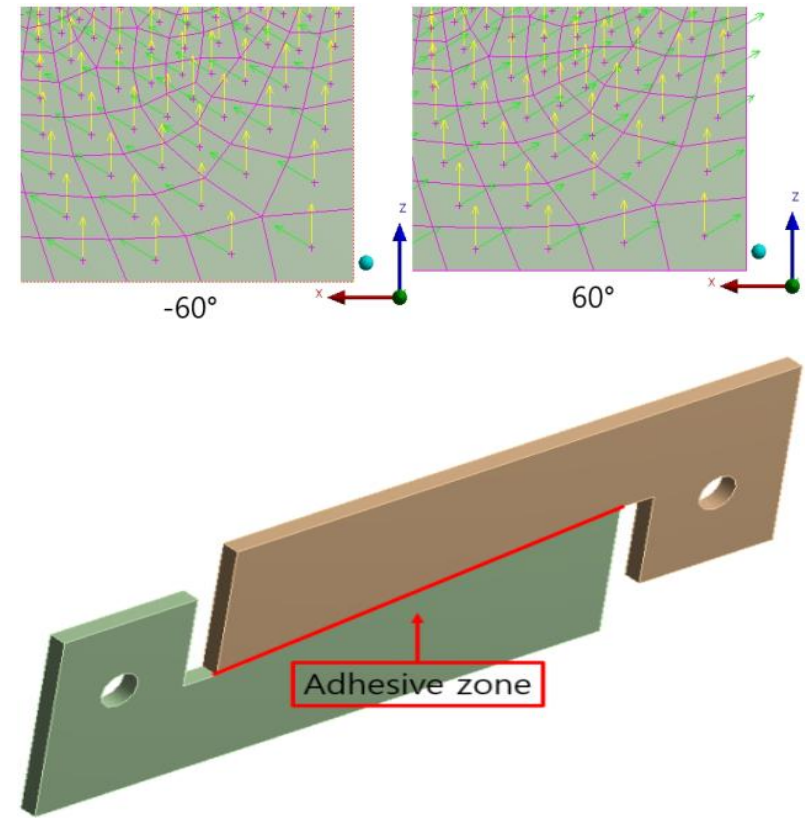

Fig. 2: Fiber Structure and Adhesive Part for Unidirectional CFRP Design

\section{Analysis conditions}

Figure 3 shows the analysis conditions, and a forced displacement at the speed of $3 \mathrm{~mm} / \mathrm{min}$. was given to the one of the cantilevers A constraint was given to the other cantilever. A degree-offreedom was given to all the load-bearing areas in the direction of rotation, which was conducted in order to check the data similar to the actual experiment without causing interference and penetration between the elements under analysis. All the analyses were conducted up to a maximum displacement of $50 \mathrm{~mm}$, which were giv- en in this way as the failure of adhesive may lead to lose adhesive force by one third of the length of bonded interface. Thus, by predicting the results, it is possible to reduce the analysis time by efficiently applying the analysis conditions. Figure 4 shows the mesh of model used in the analysis. Since it is a study to confirm the mechanical part at the bonded interface, the elements of the bonded interface have been constituted with a minimum of $0.5 \mathrm{~mm}$. The lattice structure of parts other than this part has been constituted larger for the efficiency of the analysis.

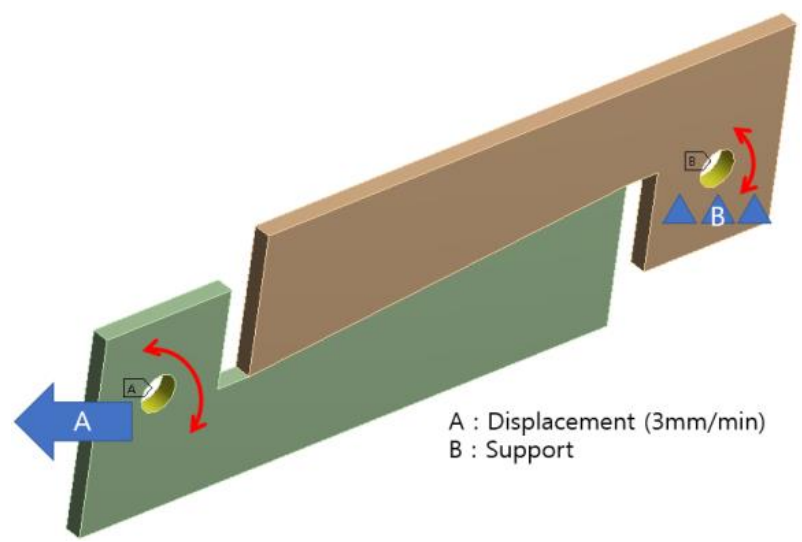

Fig. 3: Analysis Condition.

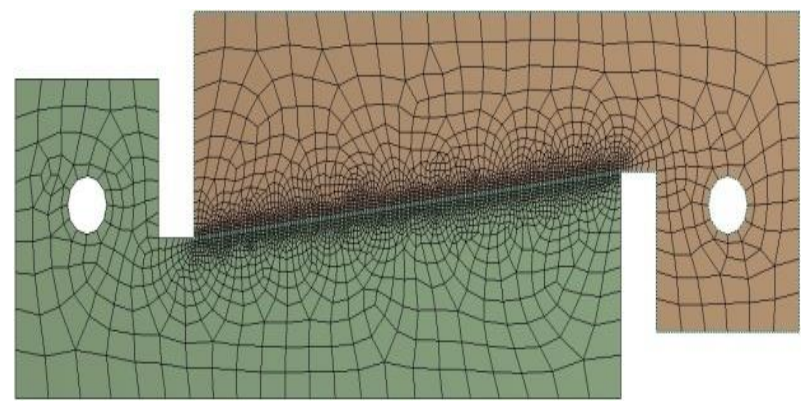

Fig. 4: Mesh Configuration of Analysis Model

\section{Simulation analysis results}

Figure 5 shows the equivalent stress distribution of TDCB 6 model. The maximum equivalent stress of TDCB6 model was about $191 \mathrm{GPa}$. Additionally, the maximum equivalent stress occurred at approximately $5 \mathrm{~mm}$ which was a somewhat early displacement. Maximum equivalent stress occurred at the midpoint of bonded interface. It is confirmed that many equivalent stresses occurred regarding the bonded interface.

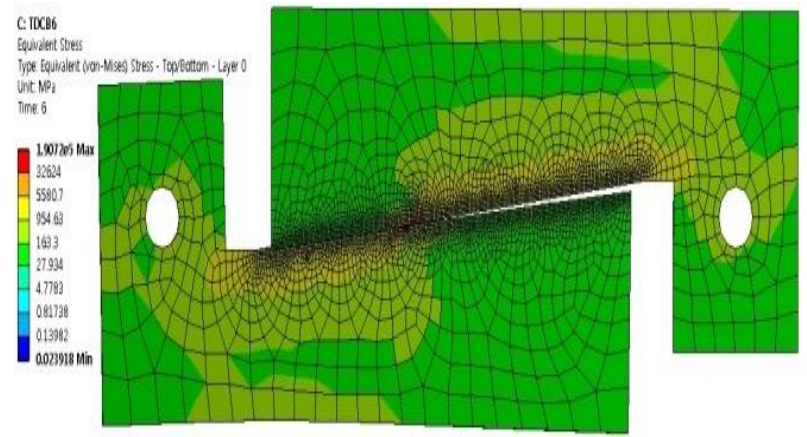

Fig. 5: Equivalent Stress Distribution of the TDCB 6 Model.

Figure 6 shows the equivalent stress distribution of TDCB 8 model. The maximum equivalent stress of TDCB8 model was about 53GPa. Like TDCB 6 model, it occurred about $5 \mathrm{~mm}$ of forced displacement. Its maximum equivalent stress is lower than that of TDCB6. The distribution of principal equivalent stress and the maximum equivalent stress also occur at the bonded interface, but 
the high equivalence stress is confirmed to occur in the neck of the research model, which is different from TDCB6 model.

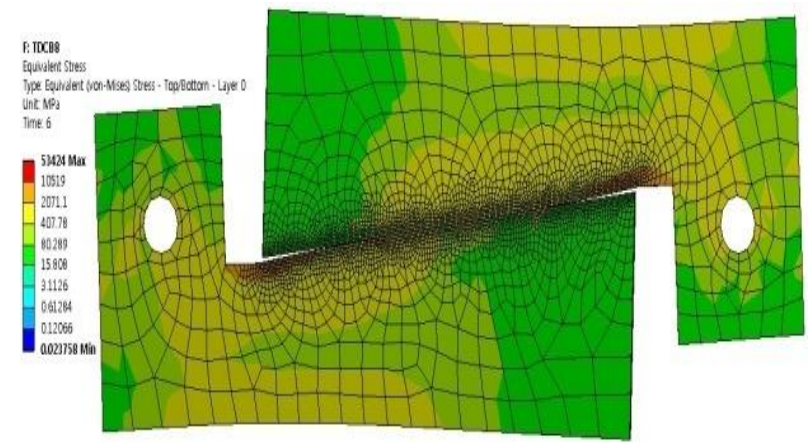

Fig. 6: Equivalent Stress Distribution of TDCB 8 Model.

Figure 7 and Figure 8 show the equivalent stresses of TDCB 10 model and TDCB 12 model. Similarities can be noticed from the equivalent stress distribution between TDCB 10 model and TDCB 12 model. Maximum equivalent stress and most equivalent stress are noticed in the neck of the research model. Moreover, it is confirmed to undergo the large forced displacement compared to the previous models. The maximum equivalent stress of TDCB 10 model is about $31 \mathrm{MPa}$ and that of TDCB 12 model is about 35 MPa. TDCB 10 and TDCB 12 models show the maximum equivalent stress lower than those of the previous models. The maximum equivalent stress of TDCB10 model occurred at the forced displacement of $50 \mathrm{~mm}$ and that of TDCB12 model at about $30 \mathrm{~mm}$.

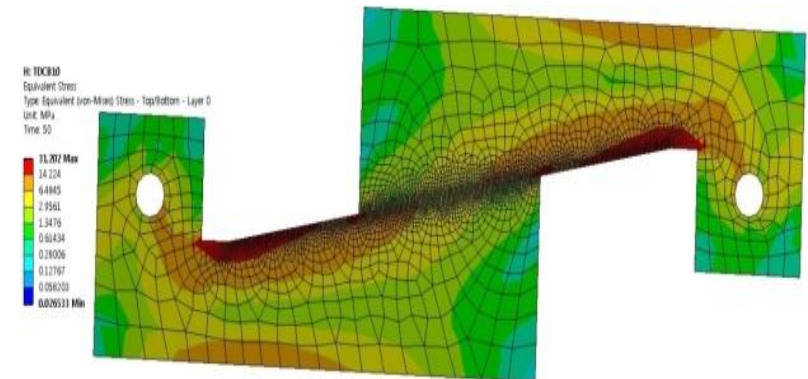

Fig. 7: Equivalent Stress Distribution of the TDCB 10 Model.

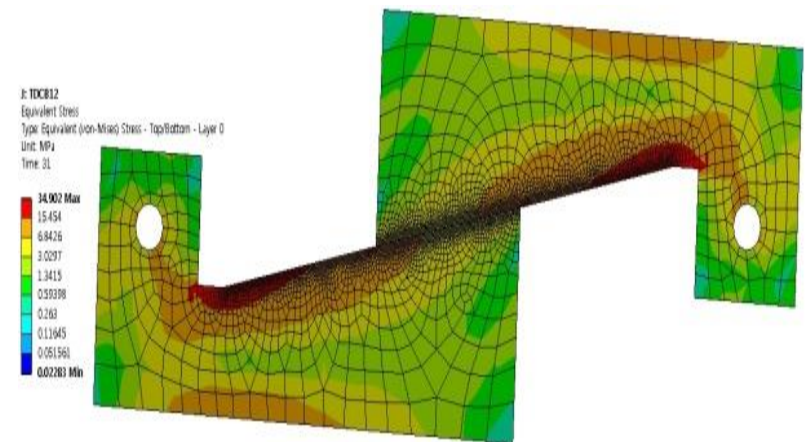

Fig. 8: Equivalent Stress Distribution of the TDCB 12 Model.

Figure 9 shows the strain distribution for all models. All models are revealed to have similar distributions of equivalent stress and strain. Maximum deformation point and maximum intensity point are the same. The maximum strains of TDCB 6 , TDCB 8 , TDCB 10 , and TDCB 12 models were revealed to be $5 \mathrm{~mm} / \mathrm{mm}$, $2 \mathrm{~mm} / \mathrm{mm}, 0.0007 \mathrm{~mm} / \mathrm{mm}, 0.0008 \mathrm{~mm} / \mathrm{mm}$, respectively. Forced displacement points at which maximum strain of all models occur are also the same as in the cases of the maximum equivalent stress.
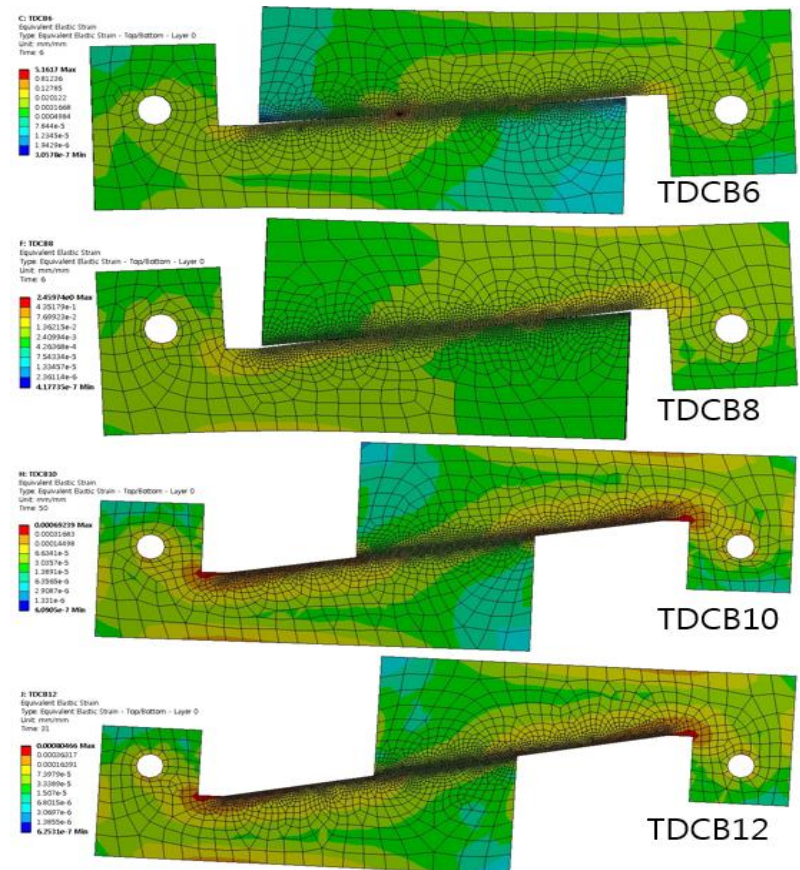

Fig. 9: Strain Distribution of Each Model.

Figure 10 is about the graph of the reaction forces of TDCB 6 and TDCB 8 models. It is the reaction force in accordance with displacement (forced displacement). Maximum reaction force occurred at the displacement of about $5 \mathrm{~mm}$ to $6 \mathrm{~mm}$ in both TDCB 6 and TDCB 8 models. Maximum reaction force of TDCB 6 model is about $80 \mathrm{KN}$. Maximum reaction force of TDCB 8 model is about $70 \mathrm{KN}$. After the maximum reaction force, reaction forces are sharply lowered to $0 \mathrm{~N}$ in both models. The point at which maximum reaction force occurs is the same as the point at which the maximum equivalent stress occurs. Also, the reaction forces of both models sharply increase at the onset of forced displacement and then fall sharply, which can be thought that the sudden destruction had occurred at the onset of displacement before complete destruction.

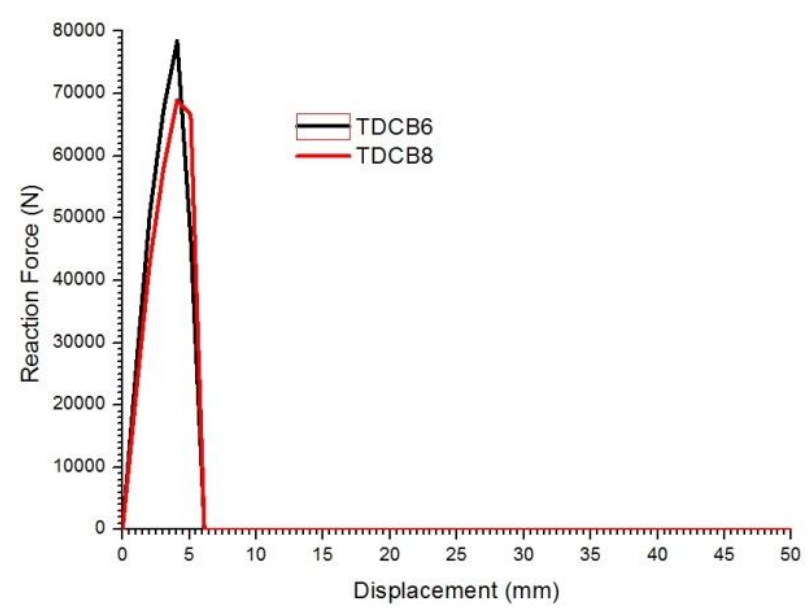

Fig. 10: Reaction Force Graph by Forced Displacement of TDCB 6 and TDCB 8 Model.

Figure 11 is about the graph of the reaction forces of TDCB 10 and TDCB 11 models. It is reaction force in accordance with displacement (forced displacement). Both TDCB10 model and TDCB12 model show a gradual graph at the onset of displacement. TDCB 10 model shows a graph in which adhesion is sustained without complete failure. TDCB12 model shows a graph in which complete rupture occurs at a forced displacement of about $44 \mathrm{~mm}$. Maximum reaction force of TDCB 10 model is about 450 $\mathrm{KN}$. Maximum reaction force of TDCB 12 model is about 470 $\mathrm{KN}$. Both models have similar maximum reaction forces, but the maximum reaction force of TDCB10 model tends to be somewhat 
lower than that of TDCB12 model. Relatively similar graphs can be noticed in TDCB10 model and TDCB12 model. However, the difference between the TDCB 6 and TDCB 8 models and the TDCB 10 and TDCB 12 models is remarkable to an appreciable extent.

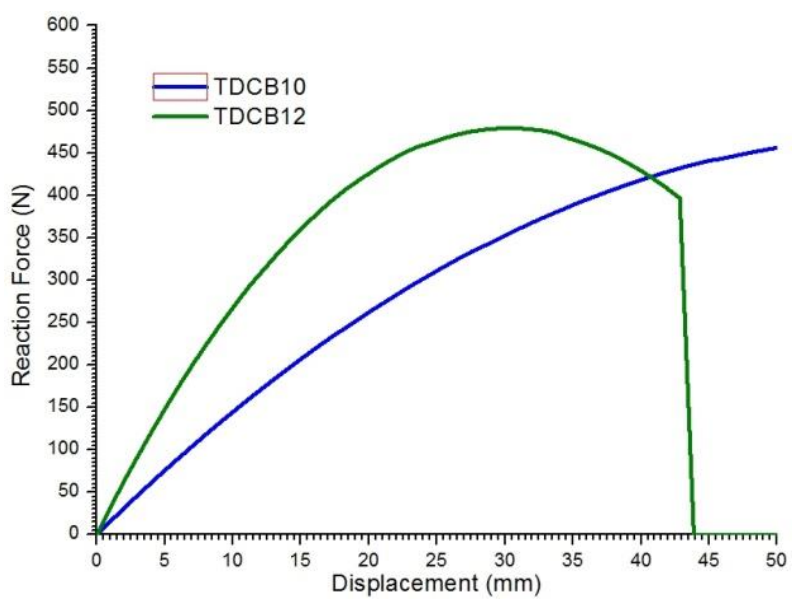

Fig. 11: Reaction Force Graph by Forced Displacement of TDCB 10 and TDCB 12 Model.

\section{Conclusion}

In this study, the mechanical properties in the sliding mode of bonded interface have been confirmed by the analyses from the finite element method using unidirectional CFRP.

1) Design of laminated structure shall be conducted in the design for the finite element analysis of unidirectional CFRP, when the laminated structure design can be unconstrainedly made to increase the degree of freedom of the user but the design of structure shall be carried out in accordance with the law of quasi-isotropic laminate structure. This is because the material designs of the composite material are so diverse that many analysis factors shall be taken into account.

2) Confirming the equivalent stress of models leads to know that the maximum equivalent stress of TDCB6 model is highest at about $191 \mathrm{GPa}$. Additionally, the maximum equivalent stress of the TDCB10 model is the lowest at about $31 \mathrm{MPa}$. However, maximum equivalent stress is revealed at the forced displacement of about $5 \mathrm{~mm}$ in TDCB6 and TDCB 8 models. Since the maximum equivalent stresses of TDCB10 model and TDCB12 model occur at the neck of the research models, the models that maintain the best adhesion at the bonded interface may be considered as TDCB 10 and TDCB 12 models. That is to say, TDCB 6 model and TDCB 8 model have a certain advantage over the other models in the structure in which a huge impact is imposed or a small deformation is not allowed. On the other hand, TDCB 10 and TDCB 12 models are suitable for the structures which are somewhat deformable under the relatively small loads. That is, TDCB6 and TDCB 8 models may be favorable in terms of adhesive stiffness and TDCB10 and TDCB12 models favorable in terms of adhesive strength.

3) It is confirmed that the equivalent stress distribution and strain distribution of all models are similar, which means the maximum deformation occurs at the maximum strength, and that the forced displacement at which the maximum strain of all models occurs are in the same position where the maximum equivalent stress occurs means that the adhesive does not slip significantly in the sliding mode. If the adhesive may be compared to the material, the maximum equivalent stress can be formed as the brittleness of material or rupture in the adhesive structure of TDCB type. Such forms are noticed remarkably in TDCB6 and TDCB8 models.
4) Compared with the TDCB6 and TDCB8 models, TDCB 10 model and TDCB 12 model have remarkably low maximum strain, which implies that all the ruptures do not occur at once but slide slowly and form and withstand the maximum equivalent stress. As this phenomenon is shown larger in TDCB6 model than inTDCB8 model, TDCB6 model is a bonding angle suitable for structures which withstand the large load or impact with a slight chance being deformed.

5) With an glance at the reaction force graphs due to the displacement of all models, TDCB 6 model and the TDCB 8 model are considered to withstand the impact loading. However, cyclic loadings cannot be sustained and it ruptures immediately. That is to say, the TDCB6 and TDCB8 models may be said to be quickly destroyed even if they withstand the large load as the forced displacement is given and the reaction force is confirmed accordingly in the analysis conditions. On the other hand, the TDCB10 and TDCB12 models show a gradual graph at the onset. In other words, the fractures of the bonding interface occur continuously but the ruptures do not occur in an easy manner. In particular, though the maximum forced displacement of 50 $\mathrm{mm}$ was given toTDCB10 model, the rupture did not occur to the last. That is, the bonding angle of $10^{\circ}$ is advantageous in bonding the structure on which impact loading is not conducted and at which a certain degree of deformation is allowed.

6) In conclusion, the advantages of sliding loading are as follows in adhering unidirectional CFRP of $60^{\circ}$ to the structures using the composite materials in accordance with the law of quasi-isotropic laminate structure. In the structure where a large sliding load shall be withstood and the deformation shall not be made to a certain degree. An angle of $6^{\circ}$ at bonded interface is favorable, and an angle of $10^{\circ}$ at bonded interface is favorable in designing the structure where little sliding load and some deformations are allowed. A bonding angle of $6^{\circ}$ may be favorable in designing the structure whose stiffness is emphasized and a bonding angle of $10^{\circ}$ in designing the structure whose strength is emphasized.

\section{Acknowledgment}

Basic Science Research Program through the National Research Foundation of Korea (NRF) funded by the Ministry of Education, Science and Technology (2015R1D1A1A01057607) supported this research.

\section{References}

[1] Blackman, B. R.K., Hadavinia,H., Kinloch, A. J., Paraschi,M.,\& Williams,J. G.. (2003).The Calculation of Adhesive Fracture Energies in Mode I: Revisiting the Tapered Double Cantilever Beam (TDCB) Test. Engineering Fracture Mechanics, 70(2), 132-141.

[2] Giorgi,M. De., Carofalo,A., Dattoma,V., Nobile,R.,\&Palano,F. (2010). Aluminum Foams Structural Modelling. Computers \& Structures, 88(1-2), 25-35.

[3] Shin,K. B.,\&Koo,D. H.. (2004). A Study on the Evaluation of the Failure for Carbody Structures Made of Laminated Fiber-reinforced Composite Materials Using Total Laminate Approach. Korean Society for Composite Materials, 17(1), 18-28.

[4] Jung,J. W., Kim,S. H., Park,J. K., \&Lee,W. I.. (2011). Research on the development of the properties of PLA composites for automatic interior parts. Korean Society for Composite Materials, 24(3), $1-5$.

[5] Hashemi,S., Kinloch,A. J.,\&Williams,J. G.. (1990). The Analysis of Interlaminar Fracture in Uniaxial Fibre-polymer Composites. Proc. R. Soc. Lond, 427(1872), 173-199.

[6] Mohamed,M., Anandan,S., Huo,Z., Birman,V., Volz,J., \&Chandrashekhara,K.. (2015). Manufacturing and characterization of polyurethane based sandwich composite structures. Composite Structures, $123,169-179$ 
[7] Boang,S. O., Kim,K. S., Kim,S. H., Song,S. G., \&Cho,J. U. (2011). Study on Compression test of Aluminum foam and honeycomb sandwich composites. Journal of the Korea AcademiaIndustrial cooperation society, 12(9), 3802-3807.

[8] Paul,A.,\& Ramamurty,U.. (2000). Strain Rate Sensitivity of a Closed-cell Aluminum Foam. Materials Science and Engineering: A, 281(1-2), 1-7.

[9] Lee, J. H., \& Cho, J. U. (2016). Evaluation and Verification on Material strength of specimen with the notches at both sides. Journal of Korean Society of Mechanical Technology, 18(5), 759-764.

[10] Bao, C., \&Cai,L.. (2011). Investigation on complian ce rotation correction for compact tensile specimen in unloading compliance method. ActaMechanica SolidaSinica, 24(2), 144-152.

[11] Katnam, K. B., Comer, A. J., Stanley, W. F., Buggy, M., \& Young, T. M. (2012). Investigating tensile behaviour of toughened epoxy paste adhesives using circumferentially notched cylindrical bulk specimens. International Journal of Adhesion \& Adhesives, 37, 3-10.

[12] Marzi,S., Biel,A.,\& Stigh,U.. (2011). on experimental methods to investigate the effect of layer thickness on the fracture behavior of adhesively bonded joints. International Journal of Adhesion and Adhesives, 31(3), 840-850. 